\title{
A FINITE ELEMENT METHOD FOR THE SOLUTION OF A POTENTIAL THEORY INTEGRAL EQUATION
}

\author{
Mark J. Rriednon \\ Lawrence Berketey Laboratory \\ One Cyctatron Roa ä \\ Berkeley, Catifomia 90720
}

Commonicated by J.C. Nedeteo

\section{$\underline{A B S T P A C P}$}

This paper discusses a finite element approximation for on integral equation of the second kind leduced from a potentiat theory boundary vatue problem in two variables. The equation is shown to admit. a unique solution, to be variatianat and coercive in the Hitbert space

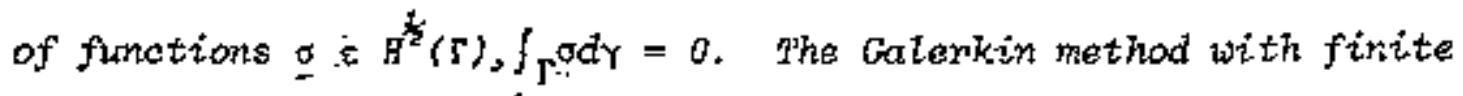
elements as trial furctions is stown to teact to an optinal rate of convergence. 
1 - LHTRODUCTIOK.

In [5], [10], the integral equation method has been used to solve the boundary valve problaw

$$
(1 . S)\left\{\begin{array}{l}
\Delta u=0 \text { in } \Omega, \\
\Delta u=0 \text { in } \Omega \cdot, \\
y-\lambda) u^{-}-(1+\lambda) u^{+}=2 g \text { on } \Gamma,|\lambda|<,, \\
\frac{\partial u^{+}}{\partial n}=\frac{\partial u^{-}}{\partial n} \text { on } T, \\
|\nabla u|=0\left(|x|^{-2}, \text { as } x \rightarrow \infty,\right.
\end{array}\right.
$$

whexe $x=\left(x_{1}, x_{2}\right), \Omega$ is a bounded domsin with a sufficiently smooth boundary curve $\Gamma$ in the plane $R_{2}, \Omega^{\prime}=R_{2} \backslash \bar{\Omega}, \bar{\Omega}=\Omega \cup \Gamma$, we denote ly $u^{*}$ time limit of $\dot{u}$ when $x$ approaches $\Gamma$ from the interior and by $u^{+}$the corresponding exterior itmit. We also adopt the convention that the normal direction of $S$ is towarts the interior of $\mathbf{\Omega}$. We denote by $\|.\|_{s},\|,\|_{s, \Omega}$, the norins in $H^{\mathrm{s}}(\Gamma), H^{\mathrm{s}}(\Omega)$ for all real $s$ (see [4] for the definition of these spsces). Let us also set $H^{s}(\Omega)=H^{5}(\Omega) \cap H_{0}^{5-\frac{1}{2}}(F)$, $H_{0}^{5}(T)=\left\{\sigma \in H^{s}(\Gamma),\langle 0,1\rangle_{H^{s}}(T) x_{H^{-s}}(\Gamma)=0\right\}, c_{0}^{1}, \alpha(\Gamma)=c^{1, \alpha}(\Gamma) \cap H_{0}^{0}(\mathrm{~S})$, where the notation $C^{1, \alpha}(O<\alpha<1)$ stands for the space of Holder continuousiy differentiable functions on $\Gamma$.

The particutar ceses of the problem (1.J) are the exterior pirkchiet problem $(\lambda=1)$ and the interior one $(\lambda=+\lambda)$. For $|\lambda|<1$, the problems of conputation of electrostatic field and magnetostatic one in piecewise bomopenequs meoid lead to this problem [10]. Let us remath that these problems acrit other formulation [10]; in [S], cho advantages of formulation $(1,1)$ for the manntostatic problens are consioered.

The solution is given in the form [5], [10], [8], of a doub]enlayex potenti.31

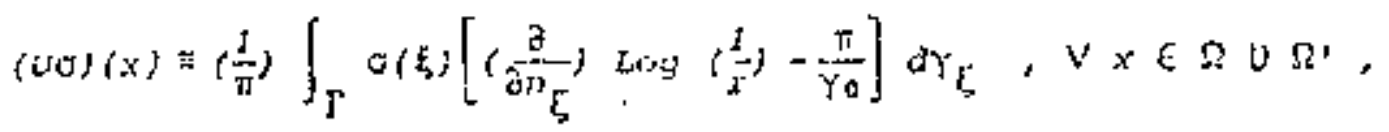


(1.3)

$$
(\mathrm{L})( \pm)=( \pm) \sigma+K \sigma,
$$

where

(1.4) (KoJ $(x)=\left(\frac{1}{\pi}\right) \int_{\Gamma} \sigma(\xi)\left(\frac{\partial}{\partial n_{\xi}}\right) \log \left[\left(\frac{J}{r}\right)-\frac{\pi}{\gamma_{0}}\right] d Y_{\xi}, \forall x \in T$.

the problem (1.1) is reduced to the Fredholm incegral egustion of the second kind in tems of the uninown ajpole density 0 on $\Gamma$

(1.5) AG $\equiv 0 * \lambda)=g$.

note that the constast $-\frac{\pi}{\gamma_{0}}$ is added in (1.2) to exclude the eigervalue $\lambda=-1$ from the spectrum of the operator $x$ [5]. In [5], [10], (1.5) has been solved numerically in $c(\Gamma)$ by a finite difference approximation.

This paper discusses a finite element approximation of (1.5)

suggested in [8]. In Section 2, we study (1.5). First, by applying the a pric: estimbtes of potential theory, we prove that (1.5) is an isomorphism in $H^{3}$ (T) for;all real $s$. Note that a similar result has been proved in [7]. Then, we introduce an operator $Q b y(2,2)$, and by using the imbeding theorens [4] and an interpolation theoren [1], we show that the inser product $(60, a)$ 。 is equivalent to the Hilbert space inner product in Ho(J). After finishing the paper, the author was informed that this result has been obtained in [7] from another point of view. Then, we shoh that $A$ is a selfugdjoint operator with respect to this inner product, and we give the variational formulation of (1.5) in fiol (I). In Section 3, we apply the Galerkin method with Einite eldents satisfying the inverse assuption (3.2) end the convergence propizit:

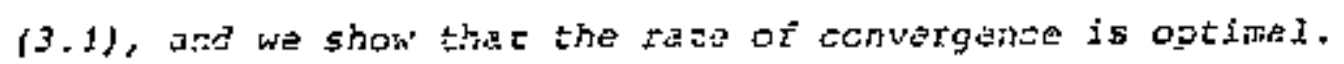

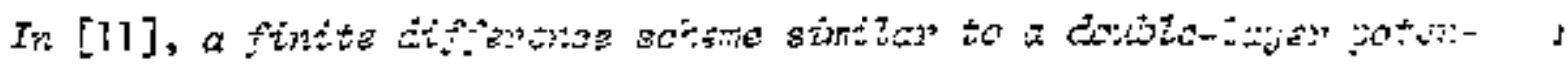

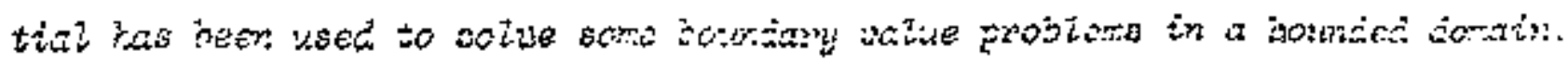
A Geleritirl method faz (1.5) (wieb $|\lambda|=f$ ) has been considerad in [7]. We

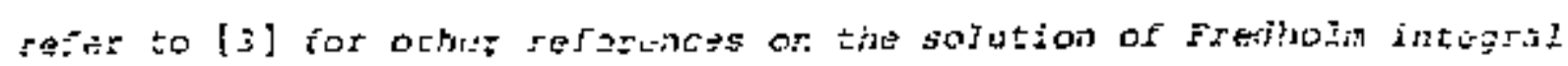

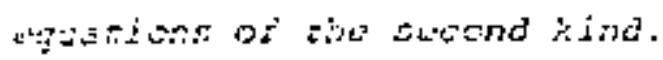

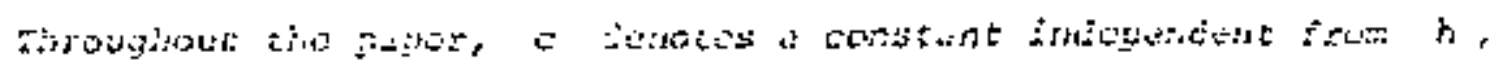


2 - VARIATIOTAL PRINCIPLE FOR THE PROBLEM IN $H_{*}^{\frac{1}{2}}(T)$.

THEOPEM 2.1 : In $H^{2}(\Gamma), s>0, K$ is carract and its spectrum is within the intervat $(-\Lambda, h), 0<\Lambda<1$. (1.5) has a imique solution in. $H^{5}(T)$ for all real $s$, and the following estimate holds :

$c_{1}\|\|_{5}<\|A\|_{3}<c_{2}\left\|_{1}\right\|_{9}$.

Proof : The operator $x$ is compact in $c(\Gamma)$ and in $h^{5}(\Gamma), s>0[3, p .458]$. For $\mu \in H^{0}(\Gamma), k \mu \in C(\Gamma)$. It follow's that the spactrom of $k$ in $H^{\mathrm{g}}(\Gamma)$. $s \geqslant 0$, is a subset of $i$ ts spectrum in $c(r)$. By [5] the matrimum absotuts value of eigenvalues of $k$ in $c(T)$ satisfies $\Lambda<. J$. Since $|\lambda|^{-1}>1,(1.5)$ has a unique solution, $\forall g \in H^{5}(T), s \geq 0$; and therefore, (2.1) is valid for $s \geqslant 0$. For $s<0$, by using a classical dvizlity argument and taking into account that $(2.1)$ holds also when $A$ is replaced by its adjoint $A^{*}$, we have

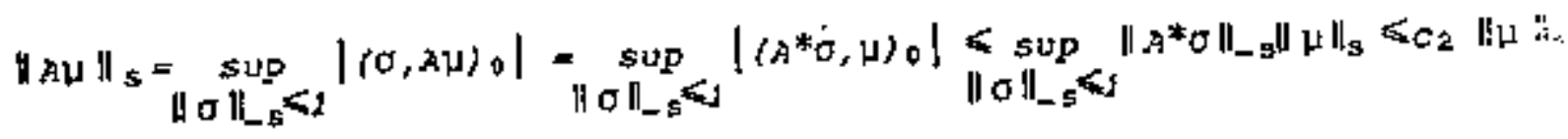
similarly, one can show the left-hand inequality in (2.1):

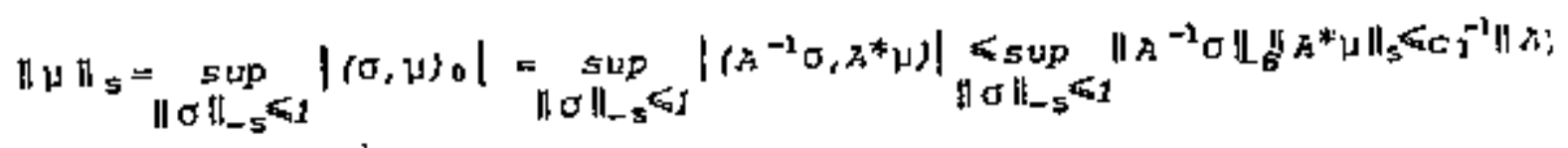
Let us define in operator $Q$ : $\infty=\frac{\partial u}{\partial n}, u=v \sigma, \forall \sigma \in c_{b}^{i}, a_{i}, i$.

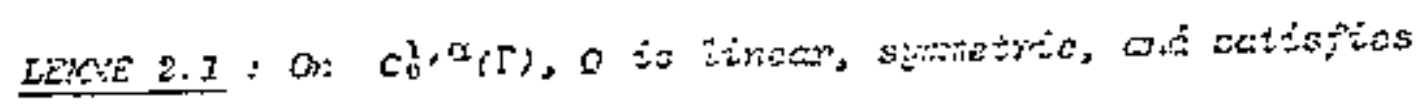

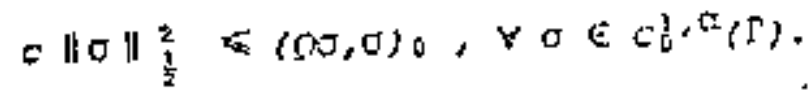

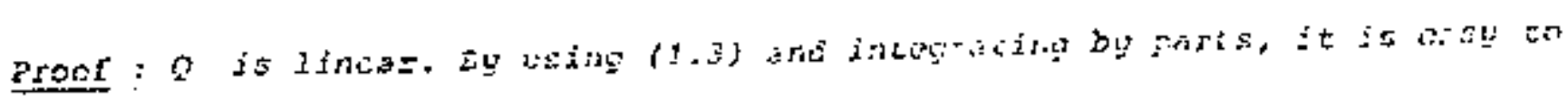

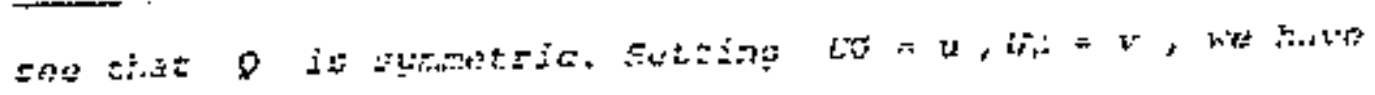


$(2.4)$

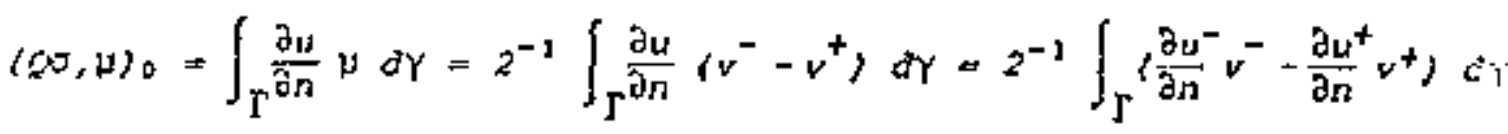

$$
\begin{aligned}
& =z^{-1} \int_{R^{2}} \nabla_{u} \nabla_{v} d x=(0,0,1)_{0} \text {. }
\end{aligned}
$$

Fdom $(2.4)$, we have

$(2,5) \quad(00,0), 22^{-1} \int_{\Omega}\left(\nabla_{u}\right)^{2} d x-$

The rorm in $H^{1}(\Omega)$ can be defined by [6]

(2.6) $\quad\|u\|_{1}^{2}, \Omega=\int_{\Omega}(\nabla u)^{2} d x+l \int_{S} u^{-} d \gamma j^{2}$

From the trace theorem [4] and Theorem 2.1 , we have for $|\lambda|=1,5=\frac{1}{2}$,

(2.7) $\quad\|u\|_{1}, \Omega>c\left\|_{u}^{-}\right\|_{\frac{1}{2}} \geqslant c,\|\sigma\|_{1}$

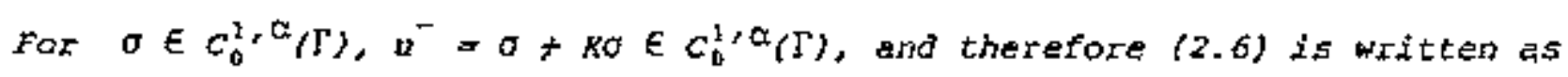

(2.8) $\|u\|_{1}, \Omega=\int_{\Omega}(\nabla u)^{2} d x, V \sigma \in c_{0}^{1}, \alpha(T)$.

Wow, (2.3) follow's from (2.5), (2.7), (2.8).

Let $H_{Q}(r)$ be the Hilbert space obtained by the completion of

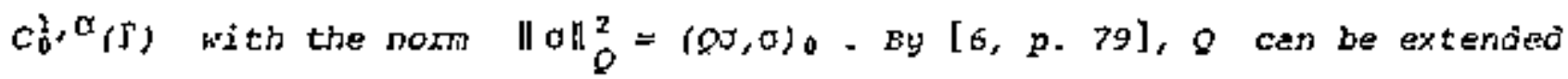
to a self-adjoint operator in $H_{Q}(S)$. Let $Q^{\frac{1}{3}}$ be its positive square root.

LE:-4 2.2 : The innex product

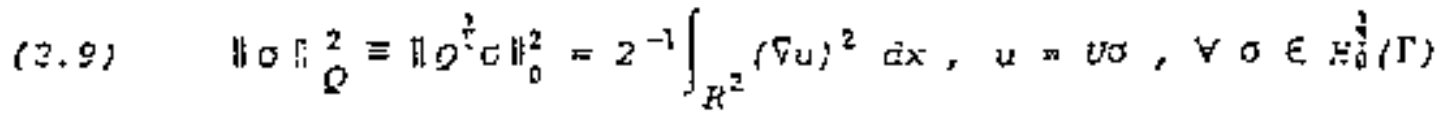

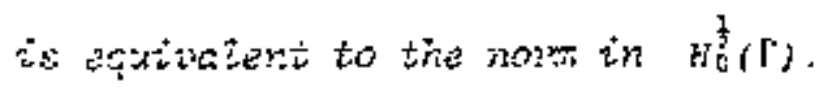

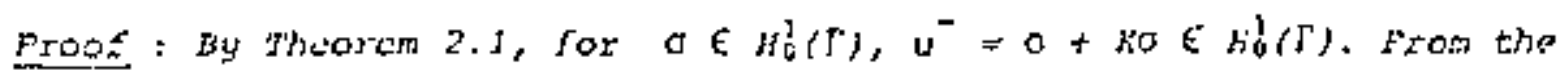

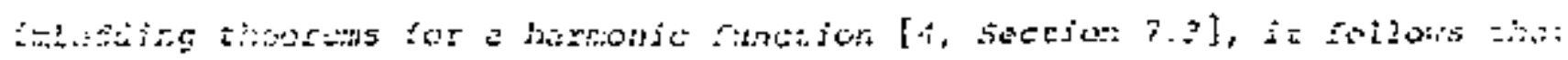

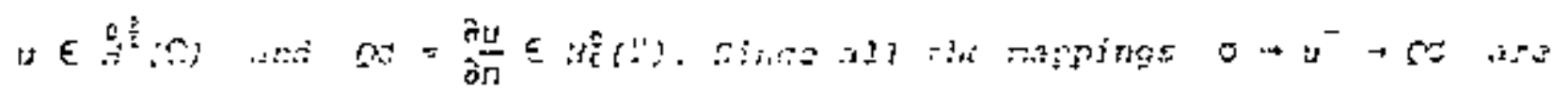

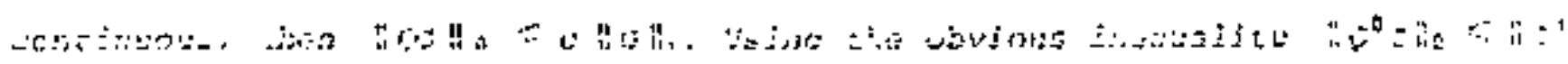


-5-

and the definition of $H^{5}(T)$; an interpolation theorem [1, p. 254] givas

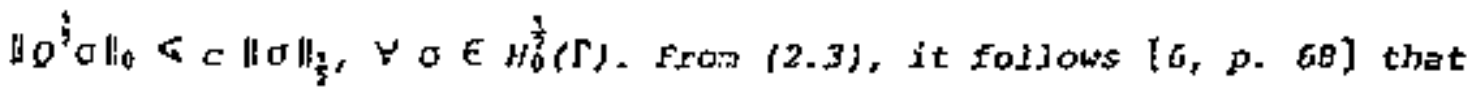

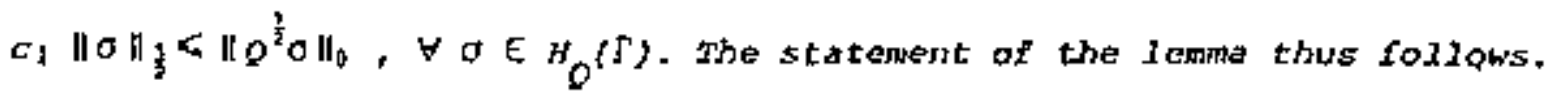

THEOPES 2.2 * A is self-adjoitit in $H_{0}^{\prime}(T)$ with respect to the imer prodict (2.9) and the following estinate holds

(2.10) $\left(1-|\lambda| N\|\mu\|_{Q}^{2} \leq a(\mu, \mu)<(t+|\lambda| A)\|\mu\|_{D}^{2}, \forall \mu \in H_{0}^{\frac{1}{2}}(\mathrm{~S}) ;\right.$ there exists a unique element of $H_{0}^{\frac{1}{2}}(\mathrm{~T})$ such that $(2, \lambda) \quad a(0, \mu)=(g, \mu)_{Q}, \forall \mu \in H_{0}^{\frac{1}{2}}(\Gamma), g \in H_{a}^{\frac{1}{2}}(\Gamma)$, where (2.52) $\quad a(0, \mu)=(A \sigma, \mu)_{Q}$. proof * Let us verify that $K$ is self-adjoint with respect to the inner product $(.,)_{O}$ - From theorem $2, J, K$ is baunded in $H_{0}^{3}(\mathrm{l})$. It is therefore sufficient to versfy that it is sprisectic for smooth $0, \mu$. By seting is $=v 0, v=v_{\mu}$ and using $(1.3)$ and $(2.4)$, we have

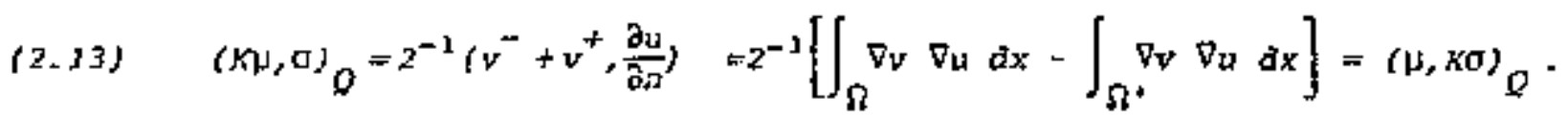

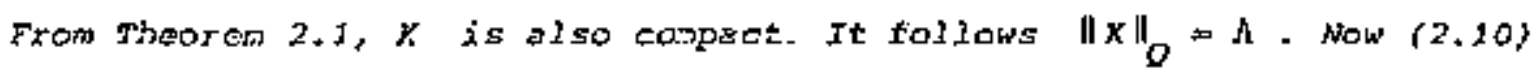
follows easily from (1.5), (2.12). The second statenent of the theorem can be deducer from the sirst cre.

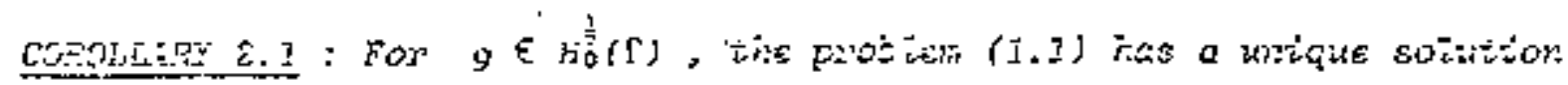
$u(x)=\left(U_{0}\right)(x), x \in R^{2} \backslash J^{4} ;|\Gamma U| \in E^{2}(\Omega) \times F^{0}(0)$.

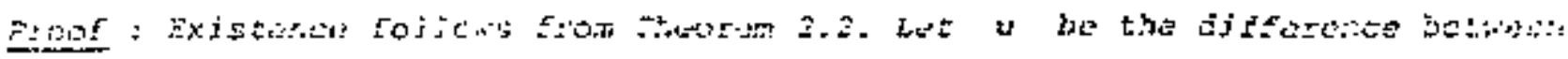
(m)

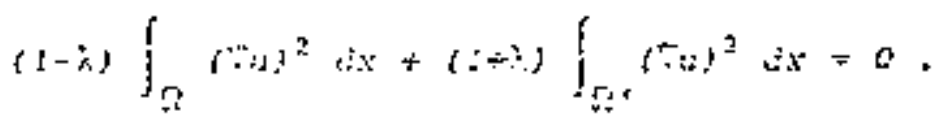


3 - THE RATE OF COHVERGEICE IN THE FIHITE ELEMENT METHOD,

$$
\text { Let } H_{h} \subset M^{k 1}(S), m>0 \text {, integer, } 0<h<1 \text {, be a regulex finite }
$$

elcment space satisfying the following conditions :

- Convergence property : $V u \in H^{3}(I), \exists \tilde{\mu}_{h} \in H_{h}$ such that for $k<\varepsilon$, with

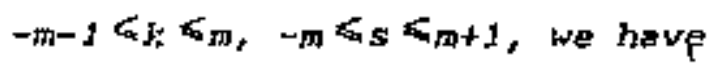

(3.1) $\quad\left\|\eta-\tilde{\mu}_{h}{ }_{k}<h^{5-k}\right\|_{h} \|_{s}$.

- Inverse assumption: $\forall \mu_{h} \in H_{h}$ for $k<5$, with $|k|,|s|<m$, we have (3.2) $\left\|\mu_{h}\right\|_{\mathrm{s}}<c h^{\mathrm{k}-5}\left\|\mu_{h}\right\|_{k}$

Remerle : The particular choice of finite elements satisfying (3.1), (3.2) it consiòered in [3].

The approximate solution $\sigma_{h}$ of $(2 . j l)$ is obtained from

$$
a\left(\sigma_{h}, \mu_{h}\right)=\left(\varphi, \mu_{h}\right)_{Q}, \forall \mu_{h} \in H_{h} .
$$

We acopt the notation $u=00, u_{h}=1 \sigma_{h}$, where o is the solution of (2.11) and $\sigma_{n}$ the solution of (3.3).

Pbeos: 3.J : Let $g \in H_{0}^{5}(T),|x|<m, k<s<x+1$. Then the error in the finitue element rethod saitisfies

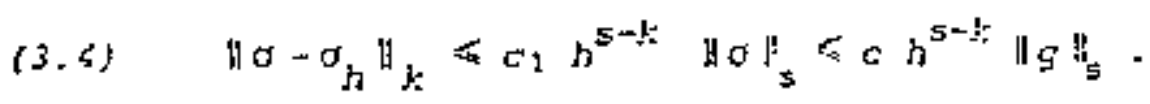

Prom:

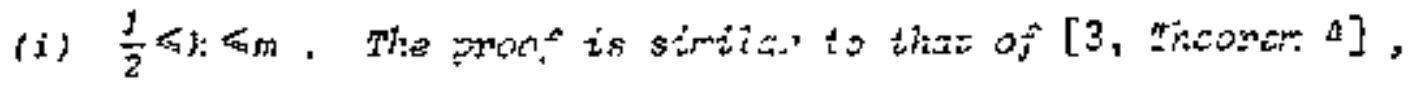

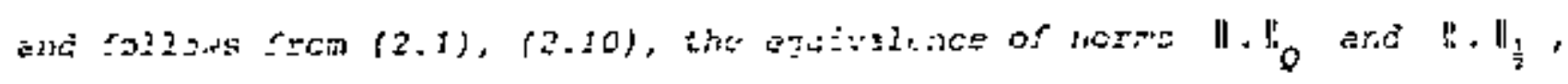
$(3.2,(3.2),(3,3)$ 
(ii) $-m<<_{\mathrm{i}}<\frac{1}{j}$. Ey using Hitocite's triek [9, pF. 166-167],

we have

$(3.5)$

$$
\left\{F, 0-\sigma_{h}{ }^{\prime} \sigma<\epsilon\left\|-\delta_{h}\right\|_{1}\left\|\sigma-\sigma_{h}\right\|_{1}, \vee \delta_{h} \in H_{h}\right. \text {. }
$$

where $r \in H_{0}^{\frac{1}{2}}(\Gamma)$, and

(3.6) $\quad a(1,0)=(2, \delta)_{Q}, \forall \delta \in \mathrm{H}_{0}^{\frac{1}{2}}(\Gamma)$.

From $(3.6),(3.1)$ for $\mathrm{k}=\frac{1}{2}$, and from Theorems $2,2,2.2$, for $\delta_{h}=\tilde{\mu}_{h}$, we ha:

(3.7) $\quad \mu-\delta_{h}\left\|_{\frac{1}{2}}<c b^{t+\frac{1}{2}}\right\| f \|_{t}, \frac{J}{2}<t<00+1$.

Substituting (3.7) and (3.4) for $k=\frac{1}{2}$ in $(3.5)$,

$$
\left(f, Q\left(\sigma-\sigma_{b}\right) J_{0}<C h^{5+t-1}\|E\|_{t}\|\sigma\|_{s}\right. \text {. }
$$

By duality in the definition of negative norns,

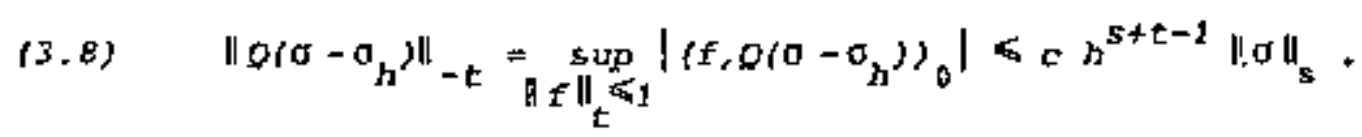

From the imbeding theorems ror a harmonic function [4, section 7.3], we

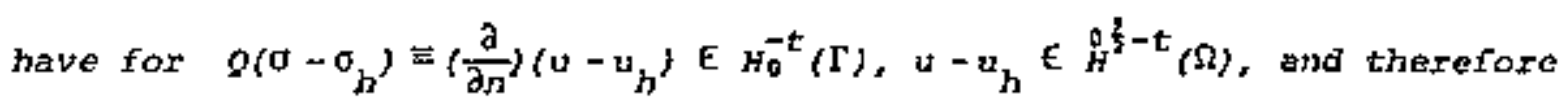
$y^{-}-u_{h}^{-} \in H_{0}^{\gamma+t}(S)$; then, by Theorem 2,1, for $|\lambda|=1, s=1-t, 0-\sigma_{b} \in H^{1-t}(\Gamma)$.

singe all the mappings $o\left(\sigma-\sigma_{h}\right) \rightarrow 4^{-}-v_{b}^{-} \rightarrow \sigma-\sigma_{h}$ are continuous, we have

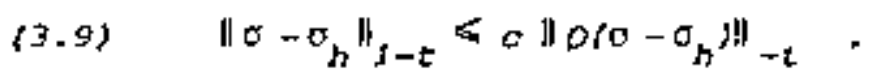

Then, (3.6) foilois Erom $(3.6)$ and (3.9).

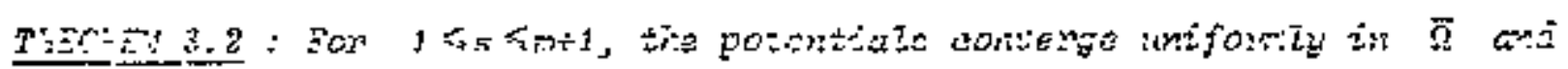
in $\overline{1}$. so that

(3.10) $\left|u(x)-u_{3}(x)\right|<0 h^{s-3}\|g\|_{3}$;

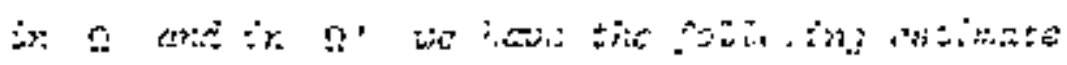

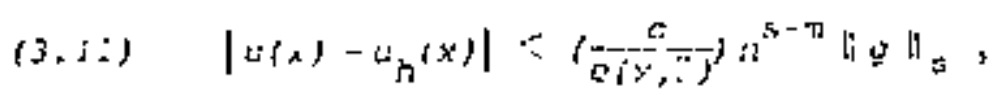

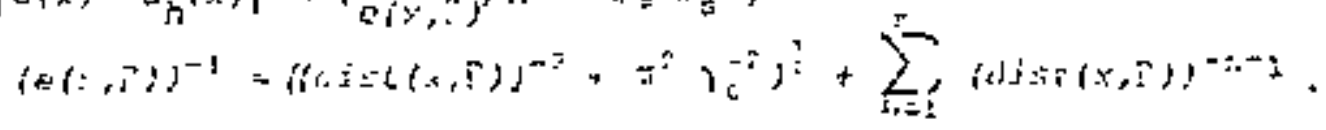


Proof : From the maxiruan prineiple,

$$
\max _{\bar{\Omega}}\left|u-u_{h}\right|=\max _{\Gamma}\left|u-u_{h}\right|=\max \left|u^{-}+u_{h}^{-}\right|
$$

Ey wing the following ineguality

$$
\left.|\sigma(s)| \zeta c h^{-\frac{1}{2}}\|\sigma\|_{0}+h^{\frac{1}{2}}\|\sigma\|_{1}\right), \forall \sigma \in H^{2}(J)
$$

Theoren 2.5 for $|\lambda|=1,5=0,1$, and Theorem 3.1 for $k=0, \mathrm{~J}$, we obtain $(3.10)$ The case $x \in \widehat{\Omega}^{\prime}$ is considered in the same way (see also $[3$, Theorem $\varepsilon]$ ). (3.11) follow's from the following inequality

$$
\left|u(x)-u_{h}(x)\right| \leqslant x \quad\left\|\sigma-\sigma_{h}\right\|_{-1} \|\left(\frac{\partial}{\partial n_{\xi}}\right)\left(\log \left(\frac{1}{x}\right)-\frac{\pi}{\gamma_{0}} \|_{\text {B }}\right.
$$

and Theorem 3.1 for $k=m$ (see also [7, p. 110]).

This way of taking the problem seems to lead to rather complex coefficients to compute. Here numerical studies are reguired. In a next paper we till considex the approxination of the boundary ave to [7] and deal with thit problem. It seems useful to give a simple remark hexe. In numerical computations, when the integral operators are replaced by matrices by using some guadrature rujes, if we need to compute $A B \bar{a}$ $(A, B$ bei 'g matrices and a a vector), we conpute $A(B \bar{d}$ and not (AB) $\bar{a}$ It would ie interesting to compare our method for the solution of (1.5) a.nd the method used in [z] ano $[1]\}$.

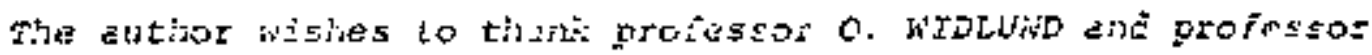

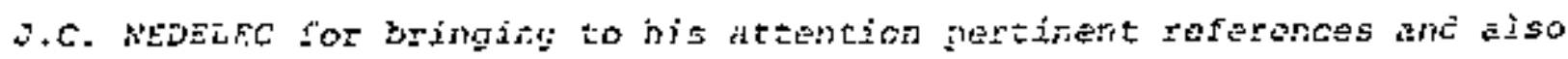
profossor I.C. MeDerec for resding the tranugrtide and giving valuble

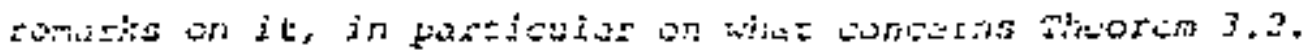


REFERENICES.

[1] EEPEzalsh, c.N., The expansion in eigenvaluefunctions of self-adjoint operntors, Naniova Dumba, Kiew, 1965 (in russian).

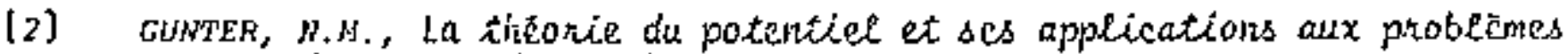
fondamentaux de La plysigue mathénatique, Paris, Gathier-viliars, 1934.

[3] H.IBO, J.C., WENDLAND, W. A finite efenent method for some integnal equations of the first find, J.N.A.A., $58(1977), 449-481$.

[4] LION, J.L., FAGENES, E., Nonhomogeneous boundaiy vatue phoblems and applications, vol. 1, Springer Veriag, Berlin, 1972.

[5] MAERGOIZ, T.D., On the numenical solution of potential theony boundary value problems by integrat equations, sib. Nat. $5 ., 12(1971)$, 1318-1326 (in russiar).

[6] MIKHLIN, S.G., Lincar partial dibferential equations, visshaya shkols, Hoscow, 1977 (in russian).

[7] NEDELEC, 2.C., Approximation des équations intëgrales en mécarique et ch phishique, Centre de Nathematiques Appliguees, Ecole Polytecilnique, g1128 palaiseau, 1977.

[8] PEKKER, M.I., On the numerical solution of lineat magnetostatic phobler.: by the conbination of the bowidary integnal method and fast lapface solver, submitted to I.E.E.E. Trans. on Nag.

[9] srRjNG, G., FIX, G., An analysis of the finitc element method, prentico Hall, New York, J973.

[10] Tozony, o.v., MARGorz, I.D., Computation of electrmagnctic bietds in three dimensions, Technika, kiev, 1974 (in russian).

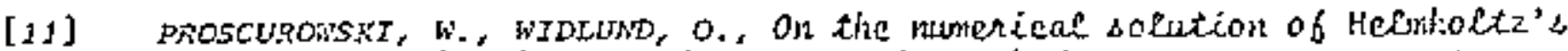
equation by the capreitence matrix method, Neth. of comp., 30 , 135 (1976), 433-368. 